\title{
Outcomes with sequential FLT3-inhibitor-based therapies in patients with AML
}

\author{
Musa Yilmaz ${ }^{1 \dagger}$, Mansour Alfayez ${ }^{1 \dagger}$, Courtney D. DiNardo ${ }^{1}$, Gautam Borthakur ${ }^{1}$, Tapan M. Kadia ${ }^{1}$, \\ Marina Y. Konopleva', Sanam Loghavi², Rashmi Kanagal-Shamanna², Keyur P. Patel'², Elias J. Jabbour', \\ Guillermo Garcia-Manero ${ }^{1}$, Naveen Pemmaraju', Sherry A. Pierce ${ }^{1}$, Issa Ghayas ${ }^{1}$, Nicholas J. Short ${ }^{1}$, \\ Guillermo Montalban-Bravo ', Koichi Takahashi', Rita Assi', Ahmad S. Alotaibi', Maro Ohanian', \\ Michael Andreeff', Jorge E. Cortes ${ }^{1}$, Hagop M. Kantarjian' ${ }^{1}$, Farhad Ravandi ${ }^{1}$ and Naval G. Daver ${ }^{1 *}$
}

\begin{abstract}
Background: Second-generation FLT3-inhibitors (FLT3i) demonstrated single-agent composite CR rates (CRC) of $45-55 \%$ in patients with relapsed/refractory (R/R) FLT3-mutated AML in phase II/III trials. However, $>85 \%$ of patients treated were prior FLT3i naïve. The response rates to sequential FLT3i exposure remain poorly defined.

Methods: We retrospectively reviewed patients with FLT3-mutated AML between November 2006 and December 2019.

Results: In frontline patients treated with a FLT3i (cohort 1), the CRC rates and median overall survival (OS) with the first $(n=56)$, second $(n=32)$, and third FLT3i-based $(n=8)$ therapy were $77 \%, 31 \%$, and $25 \%$, and 16.7 months, 6.0 months, and 1.4 months, respectively. In patients receiving a FLT3i-based therapy for the first time in a R/R AML setting (cohort 2), the CRc rates and median OS were 45\%,21\%, and 10\%, and 7.9 months, 4.0 months, and 4.1 months with the first $(n=183)$, second $(n=89)$, and third/fourth $(n=29)$ FLT3i-based therapy, respectively. In cohort 1, CRc rates with single-agent FLT3i $(n=21)$ versus FLT3i-based combinations $(n=19)$ in second/third sequential FLT3i exposures were $19 \%$ versus $42 \%$, respectively. In cohort 2 , the CRc rates with single-agent FLT3i $(n=82)$ versus FLT3i-based combinations $(n=101)$ in first FLT3i exposure were $34 \%$ versus $53 \%$, respectively, and those with single-agent FLT3i $(n=63)$ versus FLT3i-based combinations $(n=55)$ in second/third/fourth sequential FLT3i exposures were $13 \%$ versus $25 \%$, respectively.
\end{abstract}

Conclusion: CRC rates drop progressively with sequential exposure to FLT3i's in FLT3-mutated AML. In all settings, CRC rates were higher with FLT3i-based combinations compared with single-agent FLT3i therapy in similar FLT3i exposure settings.

Keyword: FLT3 mutations, Sequential FLT3 inhibitors, Midostaurin, Sorafenib, Quizartinib, Gilteritinib, FLT3-PCR, Lowintensity therapy

\footnotetext{
*Correspondence: NDaver@mdanderson.org

${ }^{\dagger}$ Musa Yilmaz and Mansour Alfayez have contributed equally to this work. 1 Department of Leukemia, The University of Texas MD Anderson Cancer Center, 1515 Holcombe Blvd, Unit FC4.2012, Houston, TX 77030, USA

Full list of author information is available at the end of the article
}

\section{Background}

Multiple tyrosine kinase inhibitors (TKIs) have demonstrated clinical activity in FLT3-mutated acute myeloid leukemia (AML), including midostaurin, sorafenib, gilteritinib, quizartinib, and crenolanib $[1,2]$. First-generation original author(s) and the source, provide a link to the Creative Commons licence, and indicate if changes were made. The images or other third party material in this article are included in the article's Creative Commons licence, unless indicated otherwise in a credit line to the material. If material is not included in the article's Creative Commons licence and your intended use is not permitted by statutory regulation or exceeds the permitted use, you will need to obtain permission directly from the copyright holder. To view a copy of this licence, visit http://creativecommons.org/licenses/by/4.0/. The Creative Commons Public Domain Dedication waiver (http://creativeco mmons.org/publicdomain/zero/1.0/) applies to the data made available in this article, unless otherwise stated in a credit line to the data. 
FLT3-inhibitors (FLT3i's) lack specificity for FLT3 (e.g. midostaurin and sorafenib), while second-generation FLT3 inhibitors (e.g. gilteritinib, quizartinib, and crenolanib) appear to be more potent and specific for FLT3, with fewer off-target effects.

The approval of first-generation FLT3i midostaurin, based on improved overall survival (OS) in the phase III RATIFY trial, established the addition of midostaurin to induction therapy as a standard approach in newly diagnosed FLT3-mutated AML [3]. Second-generation FLT3i's gilteritinib (approved in the USA and Europe for relapsed/refractory (R/R) FLT3-mutated AML based on improved OS compared with investigator choice salvage therapy in the phase III ADMIRAL trial) and quizartinib (approved in Japan based on improved OS in R/R FLT3ITD-mutated AML compared with investigator choice salvage therapy in the phase III QuANTUM-R trial) demonstrated single-agent composite complete remission $(\mathrm{CRc})$ rates $(\mathrm{CRc}=\mathrm{CR}+\mathrm{CR}$ with incomplete platelet recovery $(\mathrm{CRp})+\mathrm{CR}$ with incomplete count recovery (CRi)) of $45-55 \%$ in patients with R/R FLT3-mutated AML [4-7].

A majority (>85\%) of the R/R FLT3-mutated AML patients treated in the gilteritinib and quizartinib phase II/III trials were prior FLT3 inhibitor naïve [4-13], a population that is quickly becoming obsolete with increased testing and appropriate widespread addition of midostaurin (or sorafenib where midostaurin is not yet available) to frontline induction in patients with FLT3-mutated AML. Subset analysis from the phase II/III trials, in small numbers of R/R FLT3-mutated AML patients who had prior exposure to sorafenib or midostaurin and were subsequently treated at $R / R$ presentation with either quizartinib or gilteritinib, demonstrated lower, but still meaningful CRc rates of $25-30 \%[5,10]$. The benchmark response rates to second and even potentially third FLT3i sequential exposure, to a second-generation FLT3i in patients previously exposed to another prior second-generation FLT3i, and to single-agent FLT3i versus FLT3ibased combinations in R/R FLT3 AML, are important clinical practice and future trial development questions that remain poorly defined. We report our experience with sequential FLT3i-based therapies in patients with FLT3-mutated AML treated at our facility.

\section{Methods}

\section{Patient eligibility}

Adults ( $>/=18$ years) with frontline or R/R FLT3-ITD and/or -D835-mutated AML, who had received at least one FLT3i-based therapy at the University of Texas MD Anderson Cancer Center (UT/MDACC) between November 2006 and December 2019 were eligible. The data cutoff was May 1, 2020. Single-agent FLT3i,
FLT3i-based combinations with intensive cytotoxic chemotherapy (CCT) and with low-intensity therapy (LIT) (hypomethylating agent or low-dose cytarabinebased combinations) were included.

The most common FLT3i's (comprising 95\% of total FLT3i exposures) included were sorafenib, midostaurin, quizartinib, gilteritinib, and crenolanib (Additional file 1: Tables S3, S4). A majority of the treatments (87\%) were given on clinical trials. All clinical trials utilized are outlined in Additional file 1: Table S1. The study was conducted in accordance with the Declaration of Helsinki. All patients had signed an informed consent form approved by the Institutional Review Board (IRB). Data were collected under MDACC protocols DR09-0223 and PA12-0395 for patients with FLT3-mutated AML.

\section{Study design and objectives}

Treatment responses and $R / R$ disease were defined as per modified International Working Group criteria [14]. Our aim is to evaluate the CRc rate and survival (OS, event-free survival (EFS)) with sequential FLT3i-based therapy exposures, and to compare the CRc rates and OS, EFS with single-agent FLT3i's versus FLT3i-based combinations in similar FLT3i exposure settings. CRc $(\mathrm{CR}+\mathrm{CRp}+\mathrm{CRi})$ was as previously described in FLT3i phase III studies $[6,7,15,16]$.

Some patients received more than 1 FLT3i-based treatment during the course of their therapy. Each FLT3ibased treatment received was independently analyzed as a FLT3i-based treatment event.

Cohort 1 included patients who received their first FLT3i-based therapy in the frontline setting followed by subsequent FLT3i-based salvages. Cohort 2 included patients who received their first FLT3i-based therapy in salvage, either as the first exposure to a FLT3i in salvage or as a sequential exposure to a FLT3i in salvage. Methodologies for molecular (including multiplex polymerase chain reaction (PCR) analysis for ITD and kinase domain (D835) mutations) and multiparametric flow cytometry (MFC) assessments are in Additional file 1: Table S2.

\section{Statistical methods}

Patient characteristics were summarized using median (range) for continuous variables and frequency (percentage) for categorical variables. Categorical variables were compared for significance using the $\chi^{2}$ or Fisher's exact test, and continuous variables were analyzed using the Wilcoxon rank-sum test. OS was calculated from the date of leukemia therapy to the date of death due to any cause, censored at the last follow-up. EFS was calculated from the date of therapy to the date of disease progression, death due to any cause, or last documented followup. Kaplan-Meier method was used to estimate the 
probability of OS, and log-rank test was used to compare OS and EFS between groups of patients. Statistical analyses were performed in GraphPad and SPSS ${ }^{\odot}$ (version 24).

\section{Results}

A total of 239 patients with FLT3-ITD- and/or FLT3D835-mutated AML who received FLT3i-based treatments (including single-agent or combination FLT3i therapies) were identified (Table 1). Fifty-six patients received a FLT3i for the first time as a part of their frontline therapy (cohort 1), while 183 patients received a FLT3i for the first time as a part of their salvage therapy (cohort 2) (Fig. 1).

\section{Table 1 Clinical characteristics of patients received first FLT3i in the frontline (cohort 1) and relapse/refractory (cohort 2)} settings

\begin{tabular}{|c|c|c|}
\hline \multirow[t]{2}{*}{ Baseline clinical features } & Cohort $1(n=56)$ & Cohort $2(n=183)$ \\
\hline & \multicolumn{2}{|c|}{ Median [range], number or positive/tested (\%) } \\
\hline Age, median (range) & $62[22-90]$ & $65[21-89]$ \\
\hline Age $\geq 60$ & $30(54 \%)$ & $107(60 \%)$ \\
\hline Sex, Male & $29(51 \%)$ & $100(56 \%)$ \\
\hline SAML & $3(6 \%)$ & $44(24 \%)$ \\
\hline \multicolumn{3}{|l|}{ Karyotype } \\
\hline Diploid & $36(64 \%)$ & $68(37 \%)$ \\
\hline Monosomy 5/7 & $3(5 \%)$ & $16(9 \%)$ \\
\hline Trisomy 8 & $1(2 \%)$ & $14(8 \%)$ \\
\hline 11q23-rearrangement & $0(0)$ & $6(3 \%)$ \\
\hline Miscellaneous & $15(27 \%)$ & $65(35 \%)$ \\
\hline Insufficient metaphases & $1(2 \%)$ & $14(8 \%)$ \\
\hline \multicolumn{3}{|l|}{ Mutations } \\
\hline NPM1 & $17 / 43(39 \%)$ & $35 / 81(43 \%)$ \\
\hline DNMT3A & $10 / 22(45 \%)$ & $21 / 69(30 \%)$ \\
\hline RUNX1 & $3 / 14(21 \%)$ & $9 / 51(18 \%)$ \\
\hline TET2 & $4 / 14(29 \%)$ & $8 / 31(26 \%)$ \\
\hline WT1 & $0 / 14(0)$ & $10 / 49(20 \%)$ \\
\hline CEBPA & $3 / 20(15 \%)$ & $10 / 69(14 \%)$ \\
\hline RAS & $4 / 30(13 \%)$ & $9 / 77(12 \%)$ \\
\hline TP53 & 0/19 (0\%) & $4 / 64(6 \%)$ \\
\hline ASXL1 & $1 / 14(7 \%)$ & $6 / 43(14 \%)$ \\
\hline $\mathrm{IDH} 1$ & $1 / 22(5 \%)$ & $5 / 64(8 \%)$ \\
\hline $\mathrm{IDH} 2$ & $4 / 22(18 \%)$ & $7 / 70(10 \%)$ \\
\hline PTPN11 & $1 / 18(6 \%)$ & $4 / 64(6 \%)$ \\
\hline GATA2 & 0/14(0\%) & $1 / 49(2 \%)$ \\
\hline $\mathrm{KIT}$ & $1 / 27(4 \%)$ & $3 / 67(4 \%)$ \\
\hline Frontline therapy with a FLT3i & 56 & 0 \\
\hline $\mathrm{CCT}+\mathrm{FLT3i}$ & $33(59 \%)$ & 0 \\
\hline LIT + FLT3i & $22(39 \%)$ & 0 \\
\hline Single-agent FLT3i & $1(2 \%)$ & 0 \\
\hline Number of therapies prior to first FLT3i exposure & 0 & 2 \\
\hline Total FLT3i exposures (events) in salvage & 40 & 301 \\
\hline CCT +FLT3i & $9(22 \%)$ & $43(14 \%)$ \\
\hline LIT+FLT3i & $10(25 \%)$ & $113(38 \%)$ \\
\hline Single-agent FLT3i & $21(53 \%)$ & $145(48 \%)$ \\
\hline Total sequential FLT3i exposure (sequential events) & 40 & 118 \\
\hline
\end{tabular}

Karyotype and mutations are reported from the bone marrow prior to the first FLT3i exposure

FLT3i, FLT3 inhibitor; LIT, low-intensity chemotherapy; CCT, intensive cytotoxic chemotherapy; SAML, secondary acute myeloid leukemia 
(See figure on next page.)

Fig. 1 Cohort 1 (a) and Cohort 2 (b) patient distribution. a In cohort 1,56 patients with newly diagnosed FLT3-mutated AML received induction therapy, and 32 and 8 patients with relapsed/refractory disease received a second or third FLT3i-based therapy, respectively. Overall, 40 subsequent FLT3i exposure events were identified in cohort 1. In cohort 2, 183 patients with relapsed/refractory FLT3-mutated AML received a FLT3-inhibitor based salvage therapy, and 89, 25, and 4 patients with relapsed/refractory disease received a second, third, or fourth FLT3i-based therapy,

respectively. A total of 118 subsequent FLT3i exposure events were identified in cohort 2

\section{Cohort 1}

\section{CRc rates by salvage status and number of prior FLT3i exposures}

Fifty-six patients received FLT3i's as part of their frontline treatment: 33 with CCT, 22 with LIT, and one single-agent quizartinib. Patient characteristics are in Table 1. The CRc was 79\% (26/33) for frontline CCT with FLT3i's: 23 of 33 with sorafenib, 8 with midostaurin, 1 with gilteritinib, and 1 with quizartinib (Table 2). The CRc was $77 \%$ (17/22) for frontline LIT with FLT3i's: 13 of 22 received quizartinib with LIT (HMA or LDAC) with a CRc of $85 \%, 6,2$, and 1 received sorafenib, gilteritinib, and midostaurin-based LITs with CRc rates of $50 \%, 100 \%$, and $100 \%$, respectively. Only one treatment naïve patient received single-agent quizartinib with no response.

Among 56 patients who received a frontline FLT3i, 32 eventually relapsed or were refractory. All 32 received a second sequential FLT3i, and 8 went on to receive a third sequential FLT3i exposure for a total of 40 sequential FLT3i-based therapies in cohort 1 (Table 2).

The CRc rate in the $32 \mathrm{~s}$ FLT3i exposures was $31 \%$. CRc rates with CCT $(n=8)$, LIT $(n=9)$, and singleagent $(n=15)$ FLT3i-based therapies were $50 \%, 44 \%$, and $13 \%(P=0.118)$, respectively, suggesting a trend for benefit with combinatorial approaches in patients failing a frontline FLT3i-based therapy (Table 2). Eight of the 32 patients went on to receive a third FLT3i exposure with CRc in 2 of 8: two received a CCT- and LITbased combination, respectively, and did not respond, while 6 received single-agent FLT3i therapies on available clinical trials including 2 who received quizartinib with CRc in both, and 4 others ( 2 crenolanib, 1 AP24534, 1 E6201) with no responses (Additional file 1: Table S4B). No patient received a fourth FLT3i-based therapy in cohort 1.

\section{Overall survival and event-free survival}

The median OS was 16.7 in the frontline (first FLT3i exposure), 6.0, and 1.4 months in second and third FLT3i exposure, respectively, in cohort $1(P<0.001)$ (Fig. 2a). The median OS with LIT-based, CCT-based, and single-agent FLT3i in the $32 \mathrm{~s}$ FLT3i exposures was 5.8 months, 15.6 months, and 6.0 months, respectively $(P<0.001)$.

\section{Outcomes in patients harboring FLT3 TKD mutations}

In cohort $1,41(80 \%), 4(12 \%)$, and $6(8 \%)$ patients had ITD, D835 TKD, and ITD/D835 TKD mutations, respectively. (An additional 5 patients with no ITD versus TKD specification were excluded for this analysis.) Of 10 patients harboring D835 TKD mutations, 7 achieved CRc (70\%): 4 received sorafenib (3 achieved CRc), 3 quizartinib ( 2 achieved CRc), and 3 midostaurin ( 2 achieved CRc) as induction FLT3i. All received respective FLT3i's in combination with CCT, except 1 patient who was treated with single-agent quizartinib and did not respond. Four patients with TKD D835 mutations received a second FLT3i-based therapy (none in third FLT3i group) with CRc in 1 (25\%).

\section{Cohort 2}

\section{CRc rates by salvage status and number of prior FLT3i exposures}

A total of 183 patients with FLT3-ITD-mutated R/R AML received a FLT3i-based treatment for the first time in a salvage setting, of whom 89 received a second, 25 a third, and only 4 a fourth sequential FLT3i-based treatment (Table 2). Patient characteristics are in Table 1.

Overall, 301 (183 first FLT3i exposure in salvage, $118 \mathrm{~s} /$ third/fourth sequential FLT3i exposure in salvage) FLT3inhibitor-based treatment exposures were identified in cohort 2 (schema in Fig. 1). All 301 exposures were included in this analysis: 145 single-agent FLT3i, 113 LIT with FLT3i, and 43 CCT with FLT3i. Overall $(n=301)$, CRc rates were $45 \%, 21 \%$, and $10 \%$ with the first, second, and third/fourth FLT3i-based therapy exposures in the salvage AML setting, respectively $(\mathrm{P}<0.001)$ (Table 2). In patients treated with single-agent FLT3i's $(n=145)$, the CRc rates declined from 34 to $14 \%$ to $6 \%$ with first, second, and third/fourth FLT3i exposures $(P=0.01)$ (Table 2). In patients treated with LIT with FLT3i's ( $n=113$ ), the CRc rates declined from 54 to $31 \%$ to $28 \%$ with the first, second, and third/fourth FLT3i exposures $(P=0.061)$. In patients treated with CCT with FLT3i $(n=43)$, the CRc rates declined from 52 to $20 \%$ to $0 \%$ with the first, second, and third/fourth FLT3i exposures $(P=0.026)$.

In the first FLT3i exposure $(n=183)$ in salvage patients, the median number of prior treatments for AML was 2 (range, 1-7); however, none included a prior FLT3i as 
a

CRc rate: $77 \%$

CRc rate: $31 \%$

CRc rate: $\mathbf{2 5 \%}$

CRc rate: $\mathbf{4 5 \%}$

CRc rate: $21 \%$

CRc rate: $12 \%$

\section{6 pts received $1^{\text {st }}$ FLT3i- \\ based therapy in the \\ frontline setting}

Relapsed/Refractory

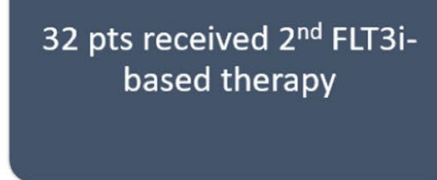

Relapsed/Refractory

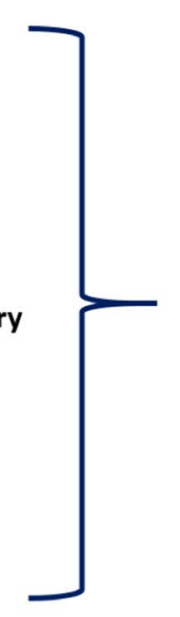

Total 40

sequential FLT3i

exposure events
183 pts received $1^{\text {st }}$

FLT3i-based therapy

in the salvage setting

Relapsed/Refractory

\section{9 pts received $2^{\text {nd }}$}

FLT3i-based therapy

\section{Relapsed/Refractory}

25 pts received $3^{\text {rd }}$

FLT3i-based therapy

\section{Relapsed/Refractory}

4 pts received $4^{\text {th }}$

FLT3i-based therapy
Total 118

sequential FLT3i

exposure events 
Table 2 Response rates by FLT3i exposure sequence

\begin{tabular}{|c|c|c|c|c|c|}
\hline \multirow[t]{2}{*}{ Therapy by cohort } & \multirow[t]{2}{*}{$\mathbf{N}$} & First FLT3i & Second FLT3i & \multirow[t]{2}{*}{ Third/fourth FLT3i } & \multirow[t]{2}{*}{$P$ value* } \\
\hline & & \multicolumn{2}{|c|}{ Number of responders/total (CRc rate) } & & \\
\hline Cohort 1 & 96 & $43 / 56(77 \%)$ & 10/32 (31\%) & $2 / 8(25 \%)$ & - \\
\hline Single-agent FLT3i & 22 & $0 / 1(0 \%)$ & 2/15 (13\%) & $2 / 6(33 \%)$ & - \\
\hline LIT+FLT3i & 32 & $17 / 22(77 \%)$ & $4 / 9(44 \%)$ & $0 / 1(0 \%)$ & - \\
\hline $\mathrm{CCT}+\mathrm{FLT3i}$ & 42 & 26/33 (79\%) & $4 / 8(50 \%)$ & $0 / 1(0 \%)$ & - \\
\hline Cohort 2 & 301 & $82 / 183(45 \%)$ & $19 / 89(21 \%)$ & $3 / 29(10 \%)$ & $<0.001$ \\
\hline Single-agent FLT3i & 145 & 28/82 (34\%) & $7 / 47(14 \%)$ & $1 / 16(6 \%)$ & 0.010 \\
\hline $\mathrm{LIT}+\mathrm{FLT3i}$ & 113 & 40/74 (54\%) & 10/32 (31\%) & $2 / 7(28 \%)$ & 0.061 \\
\hline$C C T+F L T 3 i$ & 43 & $14 / 27(52 \%)$ & $2 / 10(20 \%)$ & $0 / 6(0 \%)$ & 0.026 \\
\hline
\end{tabular}

FLT3i, FLT3 inhibitor; CRc, composite CR rate; N, number; LIT, low-intensity chemotherapy; CCT, intensive cytotoxic chemotherapy

* $P$ values added for cohort 2 patients only, as numbers of patients may be too small to make meaningful comparisons in cohort 1

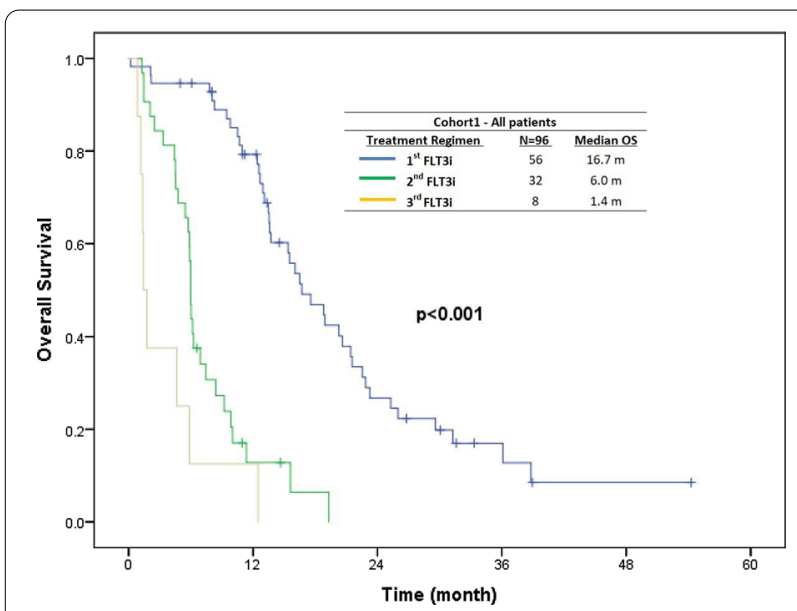

Fig. 2 The median OS of patients in cohort 1 (frontline cohort) by FLT3i exposure sequence

per definition of this cohort. CRc rates with single-agent FLT3i, LIT with FLT3i, and CCT with FLT3i were 34\%, $54 \%$, and $52 \%$, respectively $(P=0.032)$ (Table 2$)$.

In the second sequential FLT3i exposure $(n=89)$ in salvage patients, the median number of prior treatments for AML was 3 (range, 1-8), including a median of one prior FLT3i-based treatment. CRc rates with single-agent FLT3i, LIT with FLT3i, and CCT with FLT3i were 14\%, $31 \%$ and $20 \%$, respectively $(P=0.218)$ (Table 2$)$.

Only 25 patients received a third sequential FLT3ibased treatment in salvage. The median number of prior treatments for AML was 4 (range, 3-10), including a median of two prior FLT3i-based treatments. CRc rates with single-agent FLT3i, LIT with FLT3i, and CCT with FLT3i were $7 \%, 28 \%$, and $0 \%$, respectively $(P=0.255)$ (Table 2).

Only 4 patients received a fourth FLT3i-based treatment, 3 with single-agent FLT3i, and 1 in combination with CCT with no responses.

\section{CRc rates by individual FLT3i-based therapies in cohort 2}

Analyzing the data by the specific FLT3i's used, 301 independent FLT3i-based exposures were captured in cohort 2, excluding any duplication of the same FLT3i usage in any patient (Additional file 1: Table S4A). Based on the clinical trials at our institution in this time frame, the most frequently used FLT3i's in these 301 exposures were quizartinib [105 (35\%) of all exposures] and sorafenib [89 (30\%) of all exposures] (details of specific FLT3i used in Additional file 1: Table S4A).

The CRc rate with single-agent quizartinib as the first FLT3i in salvage AML $(n=46)$ was $46 \%$ (Fig. 3$)$, consistent with published phase II/III CRc rates with singleagent quizartinib in predominantly non-FLT3i-exposed patients $[7,9,13]$. The $C R c$ rate with quizartinib-based combinations as the first FLT3i exposure in salvage AML $(n=39)$ was $64 \%$ (Fig. 3 ).

In the first $(n=46)$, second $(n=9)$, and third $(n=2)$ FLT3i exposures in salvage AML, CRc rates with singleagent quizartinib progressively declined: $46 \%, 22 \%$, and $0 \%$ (Fig. 3), respectively, consistent with what has previously been presented for quizartinib [17], although numbers are small in later sequential therapies. The LIT combinations did better than single-agent quizartinib in both the first ( $n=39$ of the 46) and second ( $n=7$ of the 9) FLT3i exposures, with CRc rates of $64 \%$ and $28 \%$, respectively (Fig. 3). Only 2 patients received quizartinib with LIT in the third FLT3i exposure with one response.

Quizartinib is a potent FLT3i. We noted that switching to another FLT3i after failing quizartinib-based therapies as the first FLT3i $(n=30)$ exposure in salvage AML produced a CRc rate of only $20 \%(n=6,5$ in combination and 1 as single agent) (Additional file 1: Table S4B).

The second most common FLT3i used in salvage AML (cohort 2) was sorafenib [89 (30\%) of all exposures] (Additional file 1: Table S4A). The majority (85\%) of sorafenib therapies were administered in combination. 


\section{CRc rates with sequential FLT3i exposure in Cohort 2}

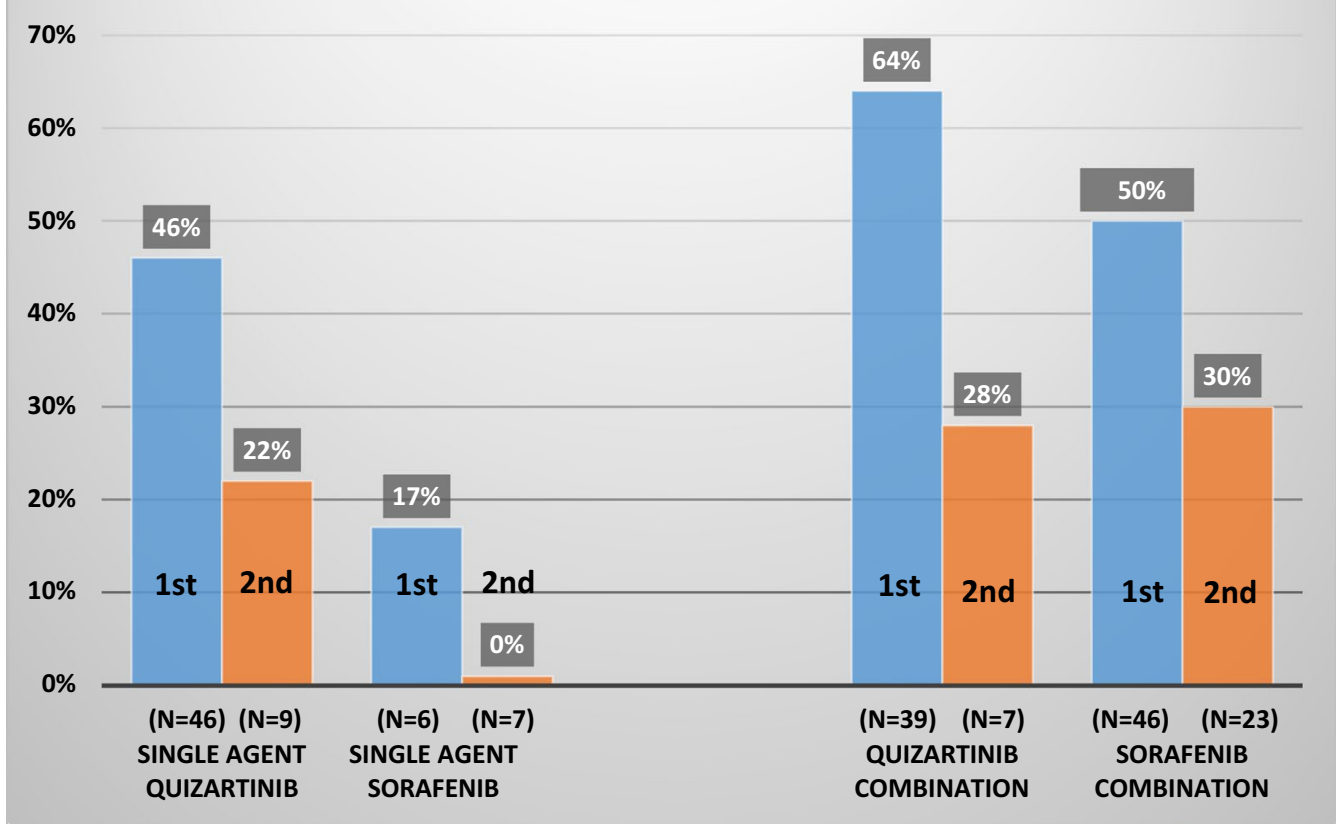

Fig. 3 First and second FLT3i exposure in cohort 2 (relapsed/refractory) as single agent and in combinations with cytotoxic chemotherapy or low-intensity treatment

CRc rates with sorafenib-based combinations in first $(n=46)$ and second $(n=23)$ FLT3i exposure settings in salvage AML were $50 \%$ and $30 \%$, respectively (Fig. 3 ), similar to previously published [18], compared with CRc rates of $17 \%$ and $0 \%$ with single-agent sorafenib in the first $(n=6)$ and second $(n=7)$ FLT3i exposure settings in salvage AML. Only 5 patients received sorafenib in combination with LIT in the third FLT3i exposure setting with 1 (20\%) CRc.

Switching to another FLT3i after failing sorafenibbased therapies as the first FLT3i $(n=35)$ in salvage was associated with a CRc rate of $17 \%$ (Additional file 1: Table S4C). Quizartinib-based therapies maintained a degree of efficacy with a CRc rate of $25 \%$ as second FLT3i-based therapies post-sorafenib failure $(n=12)$ (Additional file 1: Table S4C).

Gilteritinib was used in 13 patients in salvage in this time frame (Additional file 1: Table S4A). Eleven patients received single-agent gilteritinib, with 3 and 8, receiving it in the first and second/third FLT3i exposure settings, with respective CRc rates of $67 \%$ and $50 \%$. Two patients received gilteritinib with LIT; 1 achieved CRc. No patient received a FLT3i-based therapy after failing gilteritinib, so this could not be analyzed.
Midostaurin was always used in combination $(n=10)$, with 50\% (3 of 6) and 25\% ( 1 of 4) CRc's in the first and second FLT3i exposures in salvage, respectively (Additional file 1: Table S4A). Although the sample is small $(n=7)$, post-midostaurin CRc rates to second-generation FLT3i-based therapies were 29\% (2 of 7 responses: to quizartinib- and crenolanib-based therapies) suggesting the potential activity of second-generation FLT3i's postmidostaurin (Additional file 1: Table S4D).

Other phase 1 FLT3i-based therapies were given to 18 patients in various salvage settings, including 6 patients who received E6201, 5 FLX925, 2 KW-2449, and 1 each SAR103168, AP24534, CEP-701, TG02, and SEL25. All were used as single agent except CEP-701 (in combination with CCT). The median number of prior FLT3i exposures prior to receiving these phase 1 FLT3i's was 4 (range 1-8). No patients achieved a CRc in this group (Additional file 1: Table S4A).

\section{Overall survival and event-free survival in cohort 2}

In cohort 2 (R/R AML), the median OS was 7.9 months, 4.0 months, and 4.1 months with the first $(n=183)$, second $(n=89)$, and third/fourth $(n=29)$ FLT3i exposure, respectively $(P<0.001)$ (Fig. 4 a). Median OS with the first 


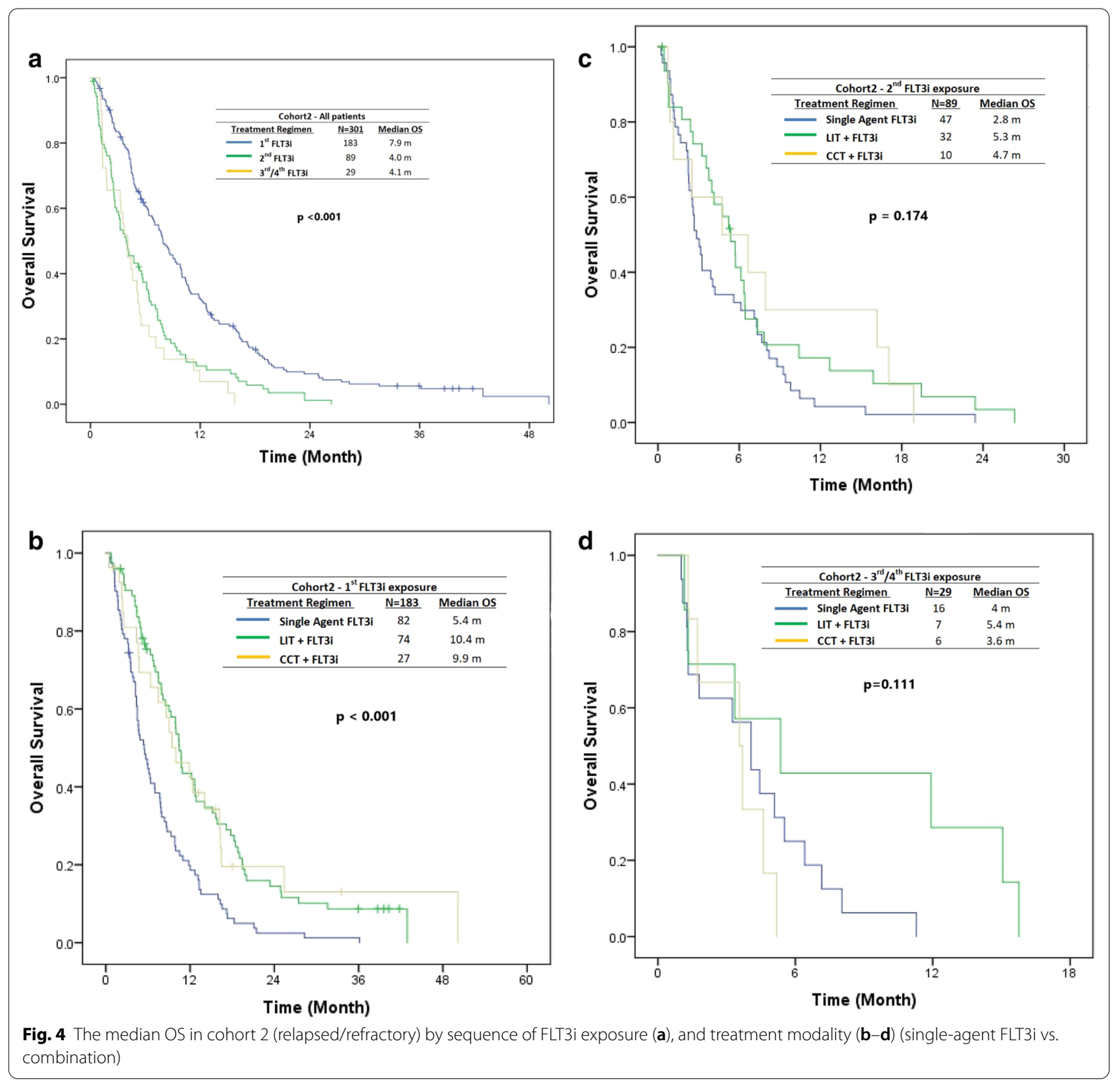

FLT3i-based therapy exposure in salvage AML was 5.4, 10.4, and 9.9 months with single-agent, LIT, and CCT FLT3i-based therapies, respectively $(P<0.001)$ (Fig. 4b). Median OS with the second FLT3i-based therapy exposure in salvage AML was 2.8, 5.3, and 4.7 months, with single-agent, LIT, and CCT FLT3i-based therapies, respectively $(P=0.174)$ (Fig. $4 \mathrm{c}$ ), implying 2.8 months compared with 5.4 months $(P=0.06)$ for single-agent versus combination therapies of FLT3i's. Median OS with the third FLT3i-based therapy exposure in salvage AML was 4.0, 5.4, and 3.6 months with single-agent, LIT, and
CCT FLT3i-based therapies, respectively $(P=0.111)$ (Fig. 4d).

\section{Outcomes in patients harboring FLT3 TKD mutations}

In cohort 2, of 183 patients, 107 (84\%), 15 (12\%), and $6(4 \%)$ patients had ITD alone, D835 TKD alone, and ITD + D835 TKD mutations, respectively. Additionally, 55 FLT3-mutated patients with no ITD versus TKD specification were excluded for this analysis. Of the 21 patients harboring D835 TKD mutations, 6 achieved CRc (30\%): 14 received crenolanib (4 achieved CRc), 3 
quizartinib (1 CRc), 2 gilteritinib (1 CRc), and 2 sorafenib (no $\mathrm{CRc}$ ) as first FLT3i. Of the 6 responses, 2 were with CCT and 4 were single-agent FLT3i. Fourteen and 6 patients harboring a TKD D835 mutations received a second and third/fourth FLT3i-based therapy with CRc in $3(21 \%)$ and $2(33 \%)$, respectively.

\section{Impact of MRD at CRc by MFC and FLT3 PCR in cohort 2 patients}

In R/R AML group (cohort 2), 104 of 301 achieved CRc, and 84 of $104(80 \%)$ of patients who achieved CRc had serial FLT3-ITD/TKD PCR checked on bone marrow at baseline and at CRc. (Median time to MRD assessment from documentation of the first CRc response was 32 days [range, 13-305 days].) Seventeen of 84 (20\%) CRc patients achieved minimal residual disease (MRD) negativity by FLT3-PCR. Patients who achieved MRD negativity by FLT3-PCR had improved OS (16.3 versus 8.5 months, $P=0.04$ ) and event-free survival (censored for transplant) (12.2 versus 3.3 months, $P<0.001)$ (Fig. 5a, b). The FLT3-PCR negativity rates are in first FLT3i exposure $(16 / 65 ; 25 \%)$, the second FLT3i exposure $(1 / 16,6 \%)$, and third/fourth FLT3i exposure settings $(0 / 3,0 \%)$.

In R/R AML group (cohort 2), 86 of $104(82 \%)$ had MRD by MFC checked on bone marrows serially at baseline and at CRc. Thirty-four of 86 (39\%) achieved
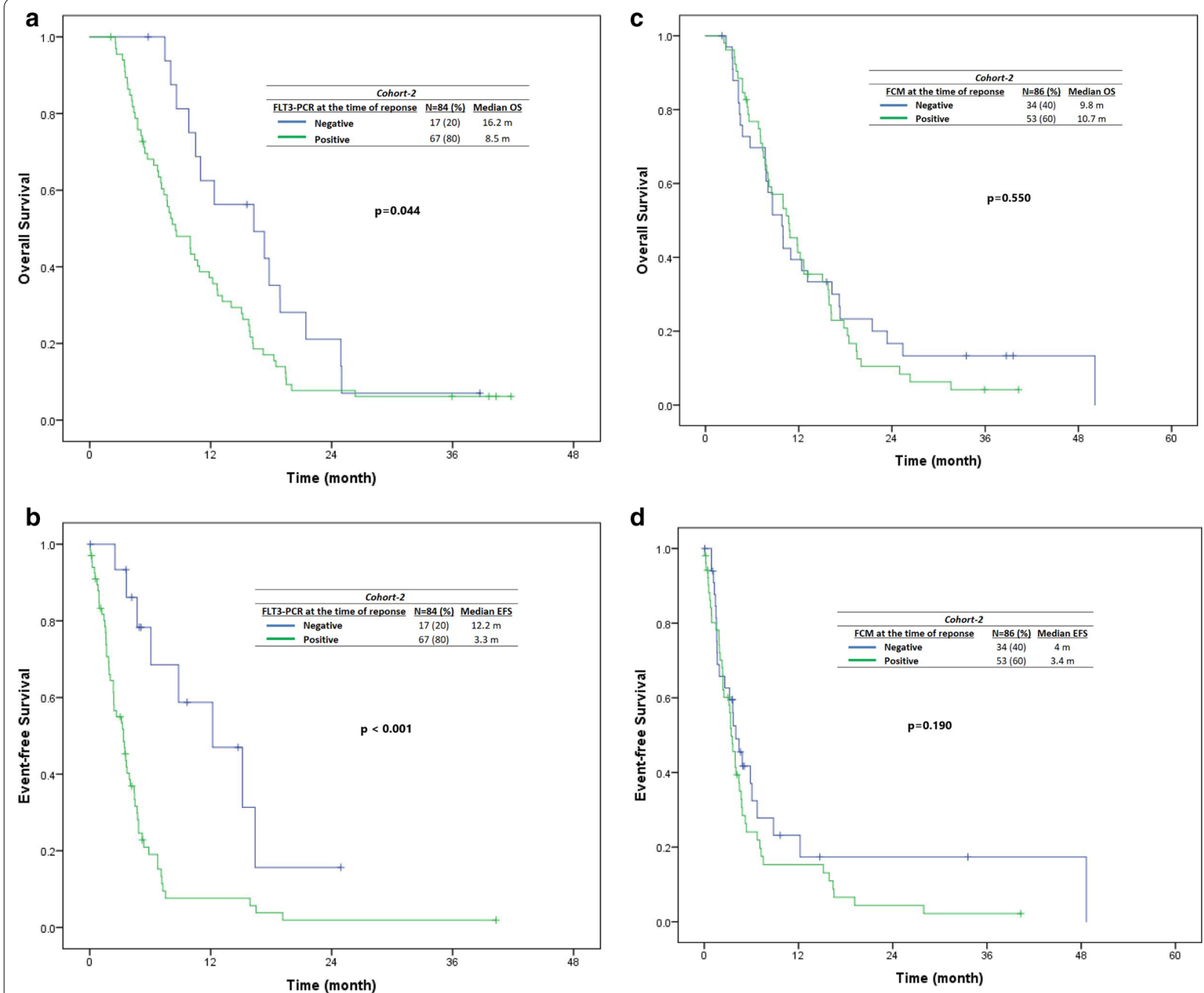

Fig. 5 Impact of minimal residual disease status on survival by FLT3-PCR [A-B] and multiparametric flow cytometry [C-D]) in cohort 2 (relapsed/ refractory) patients who achieved a CRc. In cohort 2 (relapsed/refractory), among the responders with FLT-ITD PCR checked at the time of CRC, patients who achieved minimal residual disease (MRD) negativity by FLT3 RT-PCR had improved OS and EFS (a, b). In contrast, there was no significant impact of achieving MRD negativity by multiparametric flow cytometry ( $C$ and $D$ ) on OS and EFS in R/R FLT3-mutated AML treated with FLT3i-based therapies 
MRD-negative status by MFC using the described technique. As opposed to FLT3-PCR clearance, achievement of MRD negativity by MFC at CRc was not associated with a significant impact on OS (9.8 vs 10.7 months, $P=0.55$ ) nor EFS (censored for transplant) (4 vs 3.4 months, $P=0.19$ ) (Fig. $5 \mathrm{c}, \mathrm{d}$ ). The MFC negativity rates are in first FLT3i exposure (33/67; 49\%), the second FLT3i exposure $(1 / 16,6 \%)$, and third/fourth FLT3i exposure settings $(0 / 3,0 \%)$.

We identified a correlation between MFC and PCR results. Overall, 75 patients who achieved CRc had MRD assessment performed with both MFC and PCR at response. Of 17 PCR-negative patients, 13 (76\%) were also MFC negative, and of 58 PCR-positive patients, 42 $(72 \%)$ were positive by MFC $(P=0.001)$ (Additional file 1 : Table S5).

\section{Discussion}

In our analysis, CRc rates and median OS dropped with sequential FLT3i exposure. Frontline FLT3i (midostaurin) added to induction is the recommended and widely followed approach for newly diagnosed FLT3 (ITD or D835)-mutated AML based on the phase III RATIFY results $[3,19]$. Our data suggest that expected CRc rates with sequential FLT3i-based therapies in contemporaneously treated patients will be lower $(25-31 \%)$, than the published CRc rates of $45-55 \%$, reported in phase II/ III trials of second-generation FLT3i's (gilteritinib $[5,6]$ and quizartinib $[7,9,13])$, as the majority $(85-90 \%)$ of patients on those trials (conducted prior to midostaurin approval) were prior FLT3i naive. Although lower, the CRc rates with sequential use of second-generation FLT3i's are still clinically meaningful and appeared to be higher with combinatorial approaches. These findings have direct practical implications for treating leukemia physicians.

Our findings may also be important for ongoing FLT3i clinical research. The CRc rate in combined cohorts 1 and 2 was $24 \%$ and $13 \%$ in the second $(n=29 / 121)$ and third/fourth $(n=5 / 37)$ sequential FLT3i exposure setting (single agent and combined), respectively. For singleagent FLT3i's, the CRc rates in combined cohorts 1 and 2 were lower at $15 \%$ and $14 \%$ in the second $(n=9 / 62)$ and third/fourth $(n=3 / 22)$ exposure setting, respectively (Additional file 1: Table S3, S4A). For CCT and LIT FLT3i combinations, the CRc in combined cohorts 1 and 2 was $47 \%$ and $28 \%$ in the second $(n=8 / 17,12 / 42)$ and $0 \%$ and $15 \%$ third/fourth $(n=0 / 2,2 / 13)$ exposure setting, respectively (Table 2 ). These data provide a hitherto not available benchmark for CRc rates and OS in the contemporary post-RATIFY era for evaluating second, and in the even more contemporary post-ADMIRAL era for evaluating third FLT3i-based therapy exposures. Perl A et al. recently reported a CRc rate of $88 \%$ in R/R FLT3mutated patients treated with gilteritinib and venetoclax in a phase $1 \mathrm{~B}$ study. The CRc rate was $85 \%$ in patients with $\mathrm{R} / \mathrm{R}$ FLT3-mutated AML previously exposed to FLT3i. While these data are encouraging on their own, the $85 \%$ CRc rate gains more significance when we consider that the benchmark expectations for CRc in such prior TKI-exposed patients based on this analysis would in fact be $20-30 \%$ and not $45-55 \%$ as reported in the ADMIRAL and QuANTUM-R trials. Establishing appropriate benchmarks for second and third FLT3i exposure based on the current treatment paradigm will allow us to critically analyze emerging data from ongoing trials, thereby avoiding false-negative adjudications on trials or discarding drugs/combinations that may in fact be showing encouraging activity when correctly analyzed using contemporary benchmarks.

It is important to note the difference in single-agent versus combinations. The $\mathrm{CRc}$ rates and median OS with all sequential FLT3i exposures (excluding the first FLT3i exposure) with single-agent, LIT, and CCT FLT3i-based therapies across cohorts 1 and 2 were 14\% (12/84), 33\% (16/49), and $24 \%(6 / 25)$ and $4.4 \mathrm{~m}, 7.5 \mathrm{~m}$, and $8.6 \mathrm{~m}$, respectively. While this analysis incorporates a number of different FLT3i-based single-agent and combinatorial approaches across multiple trials, which could introduce a number of potential biases, the general theme that emerges across both cohorts 1 and 2 is that combinatorial approaches may be associated with improved CRc rates and OS. In cohort 2, CRc rates with LIT and CCT were similar, $54 \%$ versus $52 \%$, respectively, in the first FLT3i exposure setting (Table 2). Although patient numbers are smaller, with second or third/fourth FLT3i exposures, CRc rates appeared to be better with LIT with FLT3i versus CCT with FLT3i regimens, 31\% versus 20\% and $28 \%$ versus $0 \%$, respectively. LIT with FLT3i appears to be feasible and effective and may be a better tolerated sequential FLT3i option compared with CCT with FLT3i, particularly in patient relapsing after a prior intensive regimen. However, randomized studies of LIT-based versus CCT-based combinations or larger single-arm studies using more homogenous LIT or CCT backbones with the same FLT3i added would be needed to draw more definitive conclusions.

Based on the clinical trials conducted at our center in the last 13-14 years (period of this analysis), the four FLT3i's commonly used in salvage, either as first FLT3i exposure or as sequential FLT3i exposure in our analysis, were sorafenib, crenolanib, quizartinib, and gilteritinib. Ravandi et al. documented a CR/CRi rate of $42 \%$ in R/R FLT3-ITD-mutated patients with azacitidine with sorafenib [18]. Among prior FLT3i-exposed patients in their study, 3 (33\%) achieved CR/CRi with azacitidine 
and sorafenib. Similarly, we noted a CRc rate of $50 \%$ in 46 FLT3-mutated R/R patients treated with sorafenib-based combinations $(\mathrm{CCT}=20$, LIT $=26)$ as first FLT3i exposure, likely with some overlap for LIT patients from the Ravandi et al. patients. Among 29 patients exposed to one prior FLT3i, 30\% (7 of 23) achieved a CRc with sorafenibbased combinations. This suggests that sorafenib-based combinations remain a reasonable option in patients with R/R FLT3 AML who have failed a prior FLT3i-based therapy as outlined in the NCCN guidelines [20].

Quizartinib has published single-agent CRc rate of $48 \%$ in the phase III QuANTUM-R study. 96\% of the patients in that study had no prior FLT3 TKI exposure. Similarly, in our analysis, the CRc rate was $46 \%$ in 46 patients who received single-agent quizartinib as the first FLT3i in salvage. CRc rates with single-agent quizartinib dropped to $22 \%$ in 9 patients who received quizartinib after exposure to one prior FLT3i. These findings are consistent with a post hoc analysis of two phase II trials of quizartinib monotherapy (NCT01565668 and NCT00989261), wherein single-agent quizartinib CRc rates were 48-53\% in prior FLT3 TKI naïve compared with $33-36 \%$ in prior FLT3 TKI-exposed patients [17]. LIT combinations with quizartinib showed high efficacy with CRc rates of $64 \%$ and $33 \%$ and median OS of 10.7 and 6.1 in prior FLT3i naïve and prior FLT3i-exposed R/R patients, respectively, consistent with previous data [21].

Gilteritinib was only used as a single-agent and in R/R setting (12 patients) during the time period analyzed. CRc rates were $67 \%$ and $38 \%$, in 3 FLT3 TKI naïve and 8 patients exposed to one prior FLT3 TKI, respectively. One additional patient received gilteritinib after two prior FLT3 TKIs and achieved CRi. These numbers are small; however, they are similar to CRc rates of $41-54 \%$ with gilteritinib monotherapy in predominantly FLT3 TKI naïve patients in published phase II/III studies, and CRc rate of $26 \%$ with gilteritinib monotherapy in a subset of 57 patients who had received a prior FLT3 TKI in the gilteritinib phase II CHRYSALIS study.

\section{Conclusion}

Second-generation FLT3i's quizartinib and gilteritinib have high activity as single-agents in patients with $R / R$ FLT3 AML who are FLT3 TKI naïve $(46-67 \%$ CRc) or exposed to one prior FLT3 TKI (22-38\% CRc). FLT3i combinations had higher response rates and improved OS compared with single-agent FLT3i's in similar FLT3i exposure settings.Numerous trials evaluating combinations of FLT3i's with induction chemotherapy, hypomethylating agents, venetoclax, and triplets of hypomethylating agents with venetoclax and FLT3i's are ongoing (NCT03661307, NCT04140487, NCT03735875) and will hopefully improve response rates and survival. These should be strongly considered for R/R FLT3-mutated AML patients, especially for patients who have failed a prior FLT3i-based therapy.

\section{Supplementary information}

Supplementary information accompanies this paper at https://doi. org/10.1186/s13045-020-00964-5.

Additional file 1. Manuscript Supplementary Information.

\section{Abbreviations}

FLT3i: FLT3 inhibitor; AML: Acute myeloid leukemia; R/R: Relapsed/refractory; CRc: Composite complete remission rate; ITD: Internal tandem duplication; IRB: Institutional Review Board; UT/MDACC: The University of Texas MD Anderson Cancer Center; CCT: Intensive cytotoxic chemotherapy; LIT: Low-intensity therapy; EFS: Event-free survival; OS: Overall survival; MFC: Multiparametric flow cytometry; HMA: Hypomethylating agent; LDAC: Low-dose cytarabine; MRD: Minimal residual disease; PCR: Polymerase chain reaction; TKI: Tyrosine kinase inhibitor; NCCN: National comprehensive cancer network; N: Number.

\section{Acknowledgements}

None.

\section{Authors' contributions}

MY, MA, and ND designed the study, analyzed the data, and wrote the paper MY, MA, SP, ND, RA, and AA collected and analyzed the data. SL, KP, and R K-S performed the molecular and cytogenetic analysis. CDD, GB, TK, MK, EJ, GGM, NP, GI, NS, G M-B, KT, MO, MA, JC, HK, and FR enrolled patients. All authors read and approved the final manuscript.

\section{Funding}

This work was supported in part by the MD Anderson Cancer Center Support Grant (CCSG) CA016672, the MD Anderson Cancer Center Leukemia SPORE

CA100632, the Charif Souki Cancer Research Fund and generous philanthropic contributions to the MD Anderson Moon Shots Program.

\section{Availability of data and materials}

The datasets used and/or analyzed during the current study are available from the corresponding author on reasonable request.

\section{Ethics approval and consent to participate}

All patients had signed an informed consent form approved by the Institutional Review Board (IRB). Data were collected under MDACC protocols DR090223 and PA12-0395 for patients with FLT3-mutated AML.

\section{Consent for publication \\ Not applicable.}

\section{Competing interests}

ND has received research funding from Daiichi Sankyo, Bristol-Myers Squibb, Pfizer, Karyopharm, Sevier, Genentech, and ImmunoGen and has served in a consulting or advisory role for Daiichi Sankyo, Bristol-Myers Squibb, Pfizer, Novartis, Celgene, AbbVie, and Agios. GB reports personal fees from FTC Therapeutics, personal fees from Argenx, personal fees from PTC Therapeutics, personal fees from BioLine Rx, personal fees from BioTheryX, personal fees from Nkarta, Inc., personal fees from Treadwell Therapeutics. JC reports grants and personal fees from Daiichi, grants and personal fees from Novartis, grants and personal fees from Pfizer, grants from Astellas, grants from Arog. CD reports personal fees from AbbVie, personal fees from Agios, personal fees from Novartis, personal fees from ImmuneOnc, personal fees from Daiichi Sankyo, personal fees from Celgene, personal fees from Takeda, personal fees from Notable Labs. Gl reports grants and personal fees from Novartis, grants from Syndax, grants from Celgene. TK reports personal fees from Novartis, grants and personal fees from Pfizer, grants from BMS, grants and personal fees from AbbVie, grants and personal fees from Genentech, grants and personal fees from JAZZ, grants from Amgen, grants from AZeneca. HK reports grants and other from AbbVie, grants and other from Agios, grants and other 
from Amgen, grants from Ariad, grants from Astex, grants from BMS, from Cyclacel, grants from Daiichi Sankyo, grants and other from ImmunoGen, grants from Jazz Pharma, grants from Novartis, grants and other from Pfizer, other from Actinium, other from Takeda. MK reports grants and personal fees from AbbVie, grants and personal fees from Genentech, grants and personal fees from F. Hoffman La Roche, grants and personal fees from Stemline Therapeutics, personal fees from Amgen, grants and personal fees from Forty -Seven, grants and personal fees from Kisoji, grants from Eli Lilly, grants from Cellectis, grants from Calithera, grants from Ablynx, grants from Agios, grants from Ascentage, grants from Astra Zeneca, grants from Kisoji, personal fees from Reata Pharmaceutical. In addition, MK has a patent US 7,795,305 B2 with royalties paid to Reata Pharma, a patent 62/993,166 issued to Eli Lilly, and a patent TBD pending to Novartis. KP reports personal fees from Astellas Pharma. NP Dr. Pemmaraju reports personal fees from Pacylex Pharmaceuticals, grants and other from Affymetrix, grants from SagerStrong Foundation, personal fees from Incyte, personal fees and other from Novartis, personal fees from LFB Biotechnologies, personal fees, non-financial support and other from Stemline Therapeutics, personal fees and non-financial support from Celgene, personal fees, non-financial support and other from AbbVie, personal fees and non-financial support from MustangBio, personal fees from Roche Diagnostics, personal fees from Blueprint Medicines, personal fees and non-financial support from DAVA Oncology, other from Samus Therapeutics, other from Cellectis, other from Daiichi Sankyo, other from Plexxikon. FR reports personal fees and other from Astellas, personal fees from Daiichi, other from Novartis. NS reports grants from Astellas, personal fees from Amgen, personal fees from AstraZeneca, grants and personal fees from Takeda Oncology. KT reports personal fees from Symbio Pharmaceuticals, Novartis, Celgene, GSK, personal fees from DAVA Oncology. MY reports grants from Daiichi Sankyo, Inc., grants from Pfizer. Rest of the authors reported no conflict of interest.

\footnotetext{
Author details

1 Department of Leukemia, The University of Texas MD Anderson Cancer Center, 1515 Holcombe Blvd, Unit FC4.2012, Houston, TX 77030, USA.

${ }^{2}$ Department of Hematopathology, The University of Texas MD Anderson Cancer Center, Houston, TX, USA.
}

Received: 27 July 2020 Accepted: 21 September 2020

Published online: 08 October 2020

\section{References}

1. Daver N, Schlenk RF, Russell NH, Levis MJ. Targeting FLT3 mutations in AML: review of current knowledge and evidence. Leukemia. 2019;33(2):299-312.

2. Swords R, Freeman C, Giles F. Targeting the FMS-like tyrosine kinase 3 in acute myeloid leukemia. Leukemia. 2012;26(10):2176-85.

3. Stone RM, Mandrekar SJ, Sanford BL, Laumann K, Geyer S, Bloomfield CD, et al. Midostaurin plus chemotherapy for acute myeloid leukemia with a FLT3 mutation. N Engl J Med. 2017;377(5):454-64.

4. Cortes JE, Tallman MS, Schiller GJ, Trone D, Gammon G, Goldberg SL, et al. Phase $2 b$ study of 2 dosing regimens of quizartinib monotherapy in. Blood. 2018a;132(6):598-607.

5. Perl AE, Altman JK, Cortes J, Smith C, Litzow M, Baer MR, et al. Selective inhibition of FLT3 by gilteritinib in relapsed or refractory acute myeloid leukaemia: a multicentre, first-in-human, open-label, phase 1-2 study. Lancet Oncol. 2017;18(8):1061-75.

6. Perl AE, Martinelli G, Cortes JE, Neubauer A, Berman E, Paolini S, et al. Gilteritinib or chemotherapy for relapsed or refractory FLT3-mutated AML. N Engl J Med. 2019;381(18):1728-40.

7. Cortes JE, Khaled S, Martinelli G, Perl AE, Ganguly S, Russell N, et al. Quizartinib versus salvage chemotherapy in relapsed or refractory FLT3ITD acute myeloid leukaemia (QUANTUM-R): a multicentre, randomised, controlled, open-label, phase 3 trial. Lancet Oncol. 2019a;20(7):984-97.

8. Cortes JE, Kantarjian H, Foran JM, Ghirdaladze D, Zodelava M, Borthakur $\mathrm{G}$, et al. Phase I study of quizartinib administered daily to patients with relapsed or refractory acute myeloid leukemia irrespective of FMSlike tyrosine kinase 3-internal tandem duplication status. J Clin Oncol. 2013;31(29):3681-7.

9. Cortes J, Perl AE, Dohner H, Kantarjian H, Martinelli G, Kovacsovics T, et al. Quizartinib, an FLT3 inhibitor, as monotherapy in patients with relapsed or refractory acute myeloid leukaemia: an open-label, multicentre, singlearm, phase 2 trial. Lancet Oncol. 2018;19(7):889-903.

10. Levis MJ, Smith CC, Ishizuka K, Kobayashi K, Arunachalam M, Wang Y, Lazzaretto D, Cortes JE. Post hoc exploratory analysis of two phase 2 trials of quizartinib monotherapy in patients (pts) with FLT3-ITD-mutated (mu) relapsed/refractory (R/R) AML with or without prior 1st-generation FLT3 tyrosine kinase inhibitors (TKI) treatment. J Clin Oncol. 2018;36:7017.

11. Cortes JE, Khaled S, Martinelli G, Perl AE, Ganguly S, Russell N, et al. Quizartinib versus salvage chemotherapy in relapsed or refractory FLT3ITD acute myeloid leukaemia (QUANTUM-R): a multicentre, randomised, controlled, open-label, phase 3 trial. Lancet Oncol. 2019b;20:986-99.

12. Perl AE. Gilteritinib significantly prolongs overall survival in patients with FLT3-mutated (FLT3mut+) relapsed/refractory (R/R) acute myeloid leukemia (AML): Results from the Phase III ADMIRAL trial. In: Proceedings of the 110th annual meeting of the American Association for Cancer Research; Atlanta, GA.: AACR; 2019.

13. Cortes JE, Tallman MS, Schiller GJ, Trone D, Gammon G, Goldberg SL, et al. Phase $2 b$ study of 2 dosing regimens of quizartinib monotherapy in FLT3ITD-mutated, relapsed or refractory AML. Blood. 2018b;132(6):598-607.

14. Cheson BD, Bennett JM, Kopecky KJ, Buchner T, Willman CL, Estey EH, et al. Revised recommendations of the International Working Group for Diagnosis, Standardization of Response Criteria, Treatment Outcomes, and Reporting Standards for Therapeutic Trials in Acute Myeloid Leukemia. J Clin Oncol. 2003;21(24):4642-9.

15. Cheson BD, Bennett JM, Kopecky KJ, Büchner T, Willman CL, Estey EH, et al. Revised recommendations of the International Working Group for Diagnosis, Standardization of Response Criteria, Treatment Outcomes, and Reporting Standards for Therapeutic Trials in Acute Myeloid Leukemia. J Clin Oncol. 2003;21(24):4642-9.

16. Döhner H, Estey E, Grimwade D, Amadori S, Appelbaum FR, BüchnerT, et al. Diagnosis and management of AML in adults: 2017 ELN recommendations from an international expert panel. Blood. 2017;129(4):424-47.

17. Levis MJ, Smith CC, Ishizuka K, Kobayashi K, Arunachalam M, Wang Y, et al. Post hoc exploratory analysis of two phase 2 trials of quizartinib monotherapy in patients (pts) with FLT3-ITD-mutated (mu) relapsed/ refractory (R/R) AML with or without prior 1st-generation FLT3 tyrosine kinase inhibitors (TKI) treatment. J Clin Oncol. 2018;36(15_suppl):7017.

18. Ravandi F, Alattar ML, Grunwald MR, Rudek MA, Rajkhowa T, Richie MA, et al. Phase 2 study of azacytidine plus sorafenib in patients with acute myeloid leukemia and FLT-3 internal tandem duplication mutation. Blood. 2013;121(23):4655-62.

19. Rollig C, Serve H, Huttmann A, Noppeney R, Muller-Tidow C, Krug U, et al. Addition of sorafenib versus placebo to standard therapy in patients aged 60 years or younger with newly diagnosed acute myeloid leukaemia (SORAML): a multicentre, phase 2, randomised controlled trial. Lancet Oncol. 2015;16(16):1691-9.

20. NCCN Clinical Practice Guidelines in AML National Comprehensive Cancer Network 2019:Version 3

21. Swaminathan M, Kantarjian H, Daver N, Borthakur G, Ohanian M, Kadia T, DiNardo C, Jain N, Estrov Z, Ferrajoli A, Garcia-Manero G, Konopleva M, Andreeff M, Pemmaraju N, Jabbour E, Alvarado Y, Wierda W, Pinsoy MR, Ravandi F, Cortes J. The combination of quizartinib with azacitidine or low dose cytarabine is highly active in patients with FLT3-ITD mutated myeloid leukemias: interim report of a phase I/II trial. Blood. 2017;128:1642.

\section{Publisher's Note}

Springer Nature remains neutral with regard to jurisdictional claims in published maps and institutional affiliations. 